\title{
A medial prefrontal cortex-nucleus acumens corticotropin-releasing factor circuitry for neuropathic pain-increased susceptibility to opioid reward
}

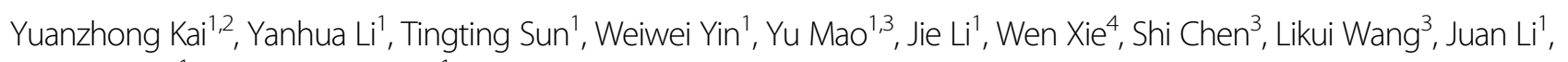
Zhi Zhang (1) and Wenjuan Tao ${ }^{1}$

\begin{abstract}
Recent studies have shown that persistent pain facilitates the response to morphine reward. However, the circuit mechanism underlying this process remains ambiguous. In this study, using chronic constriction injury (CCl) of the sciatic nerve in mice, we found that persistent neuropathic pain reduced the minimum number of morphine conditioning sessions required to induce conditioned place preference (CPP) behavior. This dose of morphine had no effect on the pain threshold. In the medial prefrontal cortex (mPFC), which is involved in both pain and emotion processing, corticotropin-releasing factor (CRF) expressing neuronal activity was increased in CCI mice. Chemogenetic inhibition of mPFC CRF neurons reversed CCl-induced morphine CPP facilitation. Furthermore, the nucleus acumens (NAc) received mPFC CRF functional projections that exerted excitatory effects on NAc neurons. Optogenetic inhibition of MPCF neuronal terminals or local infusion of the CRF receptor 1 (CRFR1) antagonist in the NAc restored the effects of neuropathic pain on morphine-induced CPP behavior, but not in normal mice. On a molecular level, in CCI mice, CRFR1 protein expression was increased in the NAc by a histone dimethyltransferase G9a-mediated epigenetic mechanism. Local G9a knockdown increased the expression of CRFR1 and mimicked CCl-induced hypersensitivity to acquiring morphine CPP. Taken together, these findings demonstrate a previously unknown and specific mPFC CRF engagement of NAc neuronal circuits, the sensitization of which facilitates behavioral responses to morphine reward in neuropathic pain states via CRFR1s.
\end{abstract}

\section{Introduction}

Non-medical abuse of prescription opioids has risen rapidly in recent years ${ }^{1-4}$, and how pain affects the likelihood of prescription opioid abuse has long been a topic of research and clinical interest ${ }^{5-8}$. However, few preclinical or clinical studies have addressed the interaction

\footnotetext{
Correspondence: Zhi Zhang (zhizhang@ustc.edu.cn) or Wenjuan Tao (wjtao01@ustc.edu.cn)

${ }^{1}$ Key Laboratory of Brain Function and Disease of Chinese Academy of Science, Department of Biophysics and Neurobiology, University of Science and Technology of China, Hefei 230027, China

${ }^{2}$ Institute of Health Sciences and technology, School of Life Sciences, Anhui University, Hefei, Anhui 2300601, China
}

Full list of author information is available at the end of the article of pain and the rewarding effects of prescription opioids. To date, the neuroanatomical and molecular substrates underlying these processes remain poorly understood.

An important link between opioid reward and chronic pain is emotional processing, as drug reward induces a positive euphoric emotion, whereas pain is associated with a negative affective state ${ }^{9-12}$. Several brain regions, such as the amygdala, thalamus, medial prefrontal cortex (mPFC), and nucleus acumens (NAc), have been implicated in both chronic pain and emotional processing ${ }^{11,13,14}$. In particular, addictive substances can alter synaptic plasticity in both the mPFC and the NAc ${ }^{15-18}$. Meanwhile, NAc and mPFC neuronal activity is necessary

\section{(c) The Author(s) 2018}

(c) (i) Open Access This article is licensed under a Creative Commons Attribution 4.0 International License, which permits use, sharing, adaptation, distribution and reproduction in any medium or format, as long as you give appropriate credit to the original author(s) and the source, provide a link to the Creative Commons license, and indicate if changes were made. The images or other third party material in this article are included in the article's Creative Commons license, unless indicated otherwise in a credit line to the material. If material is not included in the article's Creative Commons license and your intended use is not permitted by statutory regulation or exceeds the permitted use, you will need to obtain permission directly from the copyright holder. To view a copy of this license, visit http://creativecommons.org/licenses/by/4.0/. 
for the full expression of neuropathic pain-like behavior $^{19,20}$. Thus, connections involving the mPFC and NAc are most likely to be altered in chronic pain, leading to susceptibility to opioid reward ${ }^{21}$. However, the mechanisms of the cause and effect relationship between changes in neural circuitry and opioid reward have not yet been elucidated.

Corticotrophin-releasing factor (CRF), a 41-amino-acid peptide, was initially characterized as the primary neurohormone involved in controlling the body's response to stress $^{22,23}$. Later, it was found that CRF is widely expressed in the central nervous system and plays a major role not only as a stress hormone but also as a neuromodulator via the activation of the CRF type 1 receptors (CRFR1) or CRF type 2 receptors (CRFR2) 22,23 . Accumulating evidence has shown that the dysregulation of these brain CRF systems is heavily implicated in multiple psychiatric and mood disorders including drug use disorder $^{24-26}$. For example, the activation of CRFR1 in the NAc induces a positive affective state $^{22}$, and chronic CRFR1 blockage reduces heroin intake and dependenceinduced hyperalgesia ${ }^{27}$. In addition, the antagonism of CRF1Rs or the reduction of CRF1R expression attenuates hyperalgesia associated with inflammatory, visceral, and neuropathic pain in animals ${ }^{28-31}$. These findings suggest that the CRF/CRFR system may bridge the mPFC-NAc functional circuit in chronic pain-promoted susceptibility of opioid reward. To test this hypothesis, in this study, we investigated the CRFergic mPFC-NAc circuitry and molecular mechanisms underlying opioid reward facilitation under chronic neuropathic pain conditions in mice.

\section{Materials and methods \\ Animals}

In all of the experiments, C57BL/6J, G9a $\mathrm{a}^{\mathrm{f} / \mathrm{fl}}, C R F-C r e$ [strain B6(Cg)-Crhtm1(cre)Zjh/J], and $A i 9$ [strain B6. CgGt(ROSA)26Sortm9(CAG-TdTomato)Hze/J] male mice (purchased from Charles River or Jackson Laboratories) at 8-10 weeks of age were used. Except during cannula surgery, the mice were housed five per cage in a colony with ad libitum access to water and food. They were maintained under a 12-h light/dark cycle (lights on from 0700 to 1900 hours) at a stable temperature (23-25 C). All of the procedures were approved by the Care Committee of the University of Science and Technology of China.

\section{Animal model of neuropathic pain}

Mice were deeply anesthetized with isoflurane before and during CCI of the sciatic nerve or sham surgery. A small incision was made in the left thigh to expose the sciatic nerve, and three consecutive loose chromic gut ligatures (4/0) about $1 \mathrm{~mm}$ thick were placed around the nerve. For the sham surgery, the nerve was isolated without nerve ligation. All efforts were made to minimize animal suffering, to reduce the number of animals used, and to utilize alternatives to in vivo techniques. Mice that did not show pain hyperalgesia after CCI surgery were excluded from further experiments. Mechanical sensitivity was tested with a single series of von Frey filaments on the glabrous surface of each hind paw in sequence every 5-10 min. The up-down method was used to assess pain threshold.

\section{Microinjection}

We performed mPFC and NAc infusions as described previously. Before surgery, the mice were fixed in a stereotactic frame (RWD, Shenzhen, China) with a combination of xylazine $(10 \mathrm{mg} / \mathrm{kg})$ and ketamine $(100 \mathrm{mg} / \mathrm{kg})$ anesthesia. A heating pad was used to maintain mice's core body temperature at $36^{\circ} \mathrm{C}$. For the local infusion of drugs, a 26-gauge double-guide cannula (Plastics One, Roanoke, VA, USA) was implanted and aimed at the mouse $\mathrm{mPFC}$ (anteroposterior, $1.7 \mathrm{~mm}$ from the Bregma; lateral, \pm 0.25 from the midline; dorsoventral, $-3.5 \mathrm{~mm}$ from the dura) or NAc (anteroposterior, $1.5 \mathrm{~mm}$ from the Bregma; lateral, \pm 1 from the midline; dorsoventral, $-7.0 \mathrm{~mm}$ from the dura). The implanted mice were housed individually and allowed to recover from the surgery for $5 \mathrm{~d}$ before the experiments. An internal stainless steel injector, which was inserted into the guide cannula (Plastics One, Roanoke, VA, U.S) and attached to $10-\mu l$ syringes (Hamilton) with an infusion pump (Micro4, WPI, USA) at a flow rate of $100 \mathrm{nl}$ per min, was used for infusion of the vehicle $(200 \mathrm{nl}), \mathrm{CRF}$, NBI27914, or antisauvagine-30 into the nucleus. The injector was slowly withdrawn $2 \mathrm{~min}$ after the infusion. The behavioral assays were performed about $30 \mathrm{~min}$ after the infusion. Mice with missed injections were excluded.

For viral injection, 100-300 $\mathrm{nl}$ of virus (depending on its expression strength and viral titer) was injected at a rate of $30 \mathrm{nl} / \mathrm{min}$ using calibrated glass microelectrodes connected to an infusion pump. Cre-dependent virus rAAVEf1 $\alpha$-DIO-hChR2 (H134R)-mCherry-WPRE-pA (AAVChR2, AAV2 $/ 9,1.63 \times 10^{13} \mathrm{vg} / \mathrm{ml}, 200 \mathrm{nl}$ ) or rAAV-Ef1 $\alpha$ DIO-eNpHR3.0-EYFP-WPRE-pA (AAV-eNpHR3.0, $\mathrm{AAV} 2 / 9,1.18 \times 10^{13} \mathrm{vg} / \mathrm{ml}$ ) was delivered into the $\mathrm{mPFC}$ of CRF-Cre mice. 4 weeks later, the expression of mCherry was detected in the whole brain, and optogenetic manipulation was performed. rAAV-Ef1 $\alpha$ - DIO-hM3D (Gq)-mCherry-WPRE-pA (AAV-hM3Dq, AV2/8, 2.69 × $\left.10^{13} \mathrm{vg} / \mathrm{ml}\right)$ and rAAV-Ef $1 \alpha-D I O-h M 4 D(G i)-m C h e r r y-$ WPRE-pA (AAV-hM4Di, AAV2/9, $3.69 \times 10^{13} \mathrm{vg} / \mathrm{ml}$ ) were used for chemogenetic manipulations 3 weeks after injection $^{32}$. The viruses rAAV-Ef1 $\alpha$-DIO-mCherryWPRE-pA (AAV2/8, $8.93 \times 10^{12} \mathrm{vg} / \mathrm{ml}$ ) and rAAV-DIOEYFP-WPRE-pA (AAV2/9, $\left.1.95 \times 10^{12} \mathrm{vg} / \mathrm{ml}\right)$ were used 
as the controls. AAV-EF1a-mCherry-IRES-WGA-Cre (1 $\mu \mathrm{l}$, Zhien, Hefei, China) was similarly infused. Unless otherwise stated, all of these viruses were packaged by BrainVTA (Wuhan, China). All of the mice were transcardially perfused with $0.9 \%$ saline followed by ice-cold phosphate buffer $(0.1 \mathrm{M})$ containing $4 \%$ paraformaldehyde. Images of signal expressions were acquired with a confocal microscope (LSM 710, Zeiss, Germany). Animals with missed injections were excluded.

\section{Optogenetic manipulations in vivo}

An optical fiber cannula was first implanted into the NAc of an anesthetized mouse in a stereotaxic apparatus. The implants were secured to the mouse skull with dental cements. Chronically implantable fibers (diameter, $200 \mu \mathrm{m}$; Newdoon, Hangzhou) were connected to a laser generator using optic fiber sleeves. The 5-min delivery of blue light $(473 \mathrm{~nm}, 2-5 \mathrm{~mW}, 15 \mathrm{~ms}$ pulses, $20 \mathrm{~Hz})$ or yellow light (594 nm, 5-8 mW, constant) was controlled by a Master- 8 pulse stimulator (A.M.P.I., Jerusalem, Israel). The same stimulus protocol was applied to the control group of mice. Mice with missed fiber locations were excluded.

\section{Brain slice preparation}

Acute brain slices were prepared, as described in previous studies ${ }^{33}$. Mice were deeply anesthetized with pentobarbital sodium $(2 \%, w / v$, i.p. $)$ and intracardially perfused with ice-cold $\left(4^{\circ} \mathrm{C}\right)$ oxygenated modified NMDG artificial cerebrospinal fluid (NMDG ACSF) containing (in $\mathrm{mM}$ ) $93 \mathrm{~N}$-methyl-D-glucamine (NMDG), $2.5 \mathrm{KCl}, 1.2$ $\mathrm{NaH}_{2} \mathrm{PO}_{4}, 30 \mathrm{NaHCO}_{3}, 20$ HEPES, 25 glucose, 2 thiourea, $5 \mathrm{Na}$-ascorbate, $3 \mathrm{Na}$-pyruvate, $0.5 \mathrm{CaCl}_{2}, 10 \mathrm{MgSO}_{4}$, and 3 glutathione (GSH) (pH: 7.3-7.4, osmolarity: 300-305 mOsm $/ \mathrm{kg})$. Coronal slices $(300 \mathrm{~mm})$ containing mPFC or NAcon were made using a vibrating microslicer (VT1200 S, Leica, Germany), and the slices were then incubated in a submersion-type holding chamber containing oxygenated ACSF at $35^{\circ} \mathrm{C}$ throughout the experiments. Each brain slice was transferred into a slice chamber (Warner Instruments, USA) for electrophysiological recording and continuously perfused with standard perfusate at $2.5-3 \mathrm{ml} / \mathrm{min}$ at a temperature of $32^{\circ} \mathrm{C}$ maintained by an in-line solution heater (TC-344B, Warner Instruments, USA).

\section{Whole-cell patch clamp recordings}

Whole-cell patch clamp recordings were made from visually identified mPFC or NAc cells. Patch pipettes (3-5 M $\Omega$ ) were pulled from borosilicate glass capillaries (VitalSense Scientific Instruments Co., Ltd., Wuhan, China) with an outer diameter of $1.5 \mathrm{~mm}$ on a four-stage horizontal puller (P-1000, Sutter Instruments, USA). The pipettes were filled with intracellular solution containing (in $\mathrm{mM}$ ) $145 \mathrm{KsCl}, 10$ EGTA, 10 HEPES, $2 \mathrm{MgCl}_{2}, 2$
$\mathrm{CaCl}_{2}$, and $2 \mathrm{Mg}$-ATP with osmolarity adjusted to 285-290 mOsm $/ \mathrm{kg}$ and $\mathrm{pH}$ adjusted to 7.2 with $\mathrm{KOH}$. In a subset of these experiments, CRF was added to the standard ACSF, and slices were incubated in this drug solution for at least $1 \mathrm{~h}$ before the experiments began. The current-evoked firing was recorded using a current-clamp mode $(I=0 \mathrm{pA})$. The threshold current for firing was defined as the minimum strength of current injection required to elicit at least one or two spikes. The signals were acquired via a MultiClamp 700B amplifier, low-pass filtered at $2.8 \mathrm{kHz}$, digitized at $10 \mathrm{kHz}$, and analyzed with Clampfit 10.7 software (Molecular Devices, Sunnyvale, CA, USA). If the series resistance changed by $>20 \%$, the experimental recording would be terminated immediately.

\section{Light-evoked responses}

The optical stimulation was delivered using a laser (Shanghai Fiblaser Technology Co., Ltd., China) through an optical fiber $200 \mu \mathrm{m}$ in diameter positioned $0.2 \mathrm{~mm}$ away from the surface of the brain slice. To test the functional characteristics of the AAV-ChR2, the fluorescently labeled neurons expressing ChR2 from the CRF-Cre mice after 3-4 weeks virus injection were visualized and stimulated with a blue $(473 \mathrm{~nm}, 5-10 \mathrm{mV})$ laser light using a $5 \mathrm{~Hz}$, $10 \mathrm{~Hz}$, and $20 \mathrm{~Hz}$ stimulation protocol with a pulse width of $15 \mathrm{~ms}$. To test the function of CRF fibers expressing ChR2 from the mPFC, a sustained photostimulation $(473 \mathrm{~nm}, 3 \mathrm{~s}, 20 \mathrm{~Hz}, 15 \mathrm{~ms})$ was delivered in the NAc.

\section{CPP}

The general CPP procedure has been described in previous reports. In a two-chamber CPP apparatus (Huaibei Zhenhua Co., Ltd. China), the mouse was habituated and then conditioned for $30 \mathrm{~min}$ with saline or morphine in a single daily session of saline paired with one chamber in the morning and morphine paired with the other chamber in the afternoon for 3 or 5 days to induce CPP behavior. A CPP test $(15 \mathrm{~min})$ before the conditioning pre-test determined the baseline preference, and mice that spent $>60 \%$ of the total time in the chamber (equipment bias) were excluded from the study for an unbiased CPP paradigm. CPP behaviors are presented as CPP scores, defined by the formula CPP Score=time in morphine-paired chamber-time in saline-paired chamber in seconds.

\section{Western blotting}

NAc tissues were taken on day 21 after CCI surgery and were homogenized in $100 \mu \mathrm{l}$ RIPA lysis buffer with fresh protease inhibitors. The lysates were centrifuged, and the supernatant was used for SDS-PAGE. The protein concentrations were determined using a Bio-Rad protein assay kit. The protein was mixed with SDS sample buffer, heated to $95^{\circ} \mathrm{C}$ for $10 \mathrm{~min}$, separated under reducing 
conditions on an 8 or 5\% SDS-polyacrylamide gel, and transferred to a nitrocellulose membrane. Membranes were incubated in solution containing an antibody to CRFR1 (1:300, GTX130244, GeneTex), G9a (1:1000, Millipore), histone H3 dimethyl Lys9 (1:200, Active Motif), GAPDH (1:2000, Cell Signaling Technology), or $\beta-$ tubulin (1:2000, Cell Signaling Technology) with agitation overnight at $4{ }^{\circ} \mathrm{C}$. Membranes were incubated in secondary antibody to rabbit HRP (1:10000) or to mouse Ig $\operatorname{HRP}(1: 20000)$ (Calbiochem) for $1 \mathrm{~h}$ at room temperature. The bands were detected using enhanced chemiluminescence (GE Healthcare Biosciences).

\section{Chromatin immunoprecipitation (ChIP) assays}

NAc tissues were harvested and immediately crosslinked in $1 \%$ formaldehyde for $15-20 \mathrm{~min}$. After washing, the NAc tissue was homogenized for 10-30 strokes in a cell lysis buffer. The homogenate was centrifuged, and the supernatant was removed. The extracted chromatin was sheared by sonication into $200-500 \mathrm{bp}$ fragments and diluted 10-fold in ChIP dilution buffer. Ten percent of the pre-immunoprecipitated lysate was used as the "input" control for normalization. Samples were incubated with an antibody to G9a (Millipore) or H3K9me2 (Novus Biologicals). DNA and histones were dissociated with reverse buffer. Binding buffer was used for DNA precipitation and purification, and elution buffer was used to elute purified DNA from the columns. All of the buffers were provided in the ChIP kits.

\section{DNA quantification by real-time PCR}

To quantify the level of G9a and H3K9me2 at the gene promoter of CRFR1, quantitative real-time PCR was conducted with SYBR Green Master kit (Applied Biosystems) to measure the amount of G9a- and H3K9me2associated DNA, with adenine phosphoribosyltransferase (APRT, house-keeping mRNA) as the negative control. The following primers (Shenggong, Hefei, China) were used: CRFR1 (5'-CCGCTGTCTCCACTTATCTT-3' and $5^{\prime}$ - TCCCTCGTTCGTTCACTCAT-3'); APRT (5'TGCTGTTCAGGTGCGGTCAC-3' and $3^{\prime}$ AGATCCCCGAGGCTGCCTAC-3'). Each PCR reaction was repeated at least three times independently. The analysis of relative quantification of templates was performed, as described previously. Signal difference was calculated by the formula $\Delta \mathrm{Ct}=\left(\mathrm{N}_{\exp }-\mathrm{N}_{\mathrm{ave}}\right) \times \mathrm{Ct}_{\mathrm{ave}}$ $\left(\mathrm{N}_{\text {exp }}\right.$, normalized Ct value of the target or $\mathrm{Ct}_{\text {target }} / \mathrm{Ct}_{\text {input }}$; $\mathrm{N}_{\text {ave }}$, mean $\mathrm{N}$ value for control; and $\mathrm{Ct}_{\text {ave }}$, mean $\mathrm{Ct}$ value for control).

\section{Immunohistochemistry}

The mice were deeply anesthetized with pentobarbital sodium $(50 \mathrm{mg} / \mathrm{kg}$, i.p.) and sequentially perfused with saline and $4 \%(\mathrm{w} / \mathrm{v})$ paraformaldehyde (PFA). The brains were subsequently removed and post-fixed in 4\% PFA at $4{ }^{\circ} \mathrm{C}$ overnight. After cryoprotection of the brains with $30 \%(\mathrm{w} / \mathrm{v})$ sucrose, coronal sections $(40 \mu \mathrm{m})$ were cut on a cryostat (Leica CM1860) and used for immunofluorescence. The sections were incubated in $0.3 \%(\mathrm{v} / \mathrm{v})$ Triton X-100 for $0.5 \mathrm{~h}$, blocked with $10 \%$ donkey serum for $1 \mathrm{~h}$ at room temperature, and incubated with the CRF antibody, (1:1000, T-4037, Peninsula Laboratories), at $4{ }^{\circ} \mathrm{C}$ for $24 \mathrm{~h}$, followed by the corresponding fluorophoreconjugated secondary antibodies (ThermoFisher) for $2 \mathrm{~h}$ at room temperature.

\section{Statistics and drugs}

We conducted simple statistical comparisons using the paired Student's $t$-test (two-tailed). Two-way ANOVA and Bonferroni's post hoc analyses were used to statistically analyze the data from the experimental groups with multiple comparisons. All of the data are expressed as the mean \pm SEM, and significance levels are indicated as ${ }^{*} p<$ $0.05,{ }^{* * *} p<0.01$, and ${ }^{* * * *} p<0.001$. OriginPro 2017 software (OriginLab Corporation, USA) and GraphPad Prism 5 (GraphPad Software, Inc., USA) were used for statistical analyses and graphing. Offline analysis of the data obtained from electrophysiological recordings was conducted using Clampfit software version 10.7 (Axon Instruments, Inc., USA). Drugs were purchased from Sigma-Aldrich (St. Louis, MO) or Tocris Bioscience (Ellisville, MO).

\section{Results}

Neuropathic pain facilitates morphine-induced preference behavior

We used a well-accepted mouse model of neuropathic pain with a chronic constriction injury (CCI) of the sciatic nerve ${ }^{34}$. CCI-induced persistent mechanical pain sensitization, which lasted for at least 49 days after surgery (Fig. 1a). The morphine conditioned place preference (CPP) paradigm was used to determine the response to morphine reward, and we compared the rate of CPP induction by morphine conditioning sessions in $\mathrm{CCI}$ and sham mice from 21 days after surgery (Fig. 1b). In CCI mice conditioned daily with morphine at $0.3 \mathrm{mg} /$ $\mathrm{kg}$, a minimum of three conditioning sessions was required to induce significant CPP behavior; in contrast, in the sham mice, three such sessions were insufficient (Fig. 1c). Meanwhile, with five such sessions, or three sessions with $1 \mathrm{mg} / \mathrm{kg}$ morphine conditioning, CCP behavior was reliably established in the sham mice (Fig. 1d). Interestingly, $0.3 \mathrm{mg} / \mathrm{kg}$ morphine had no effect on CCI mice's pain threshold 21 days after surgery (Fig. 1e). These results suggest that persistent neuropathic pain facilitates the acquisition of morphine CPP behavior, which is not due to morphine's inhibition of pain. To confirm this hypothesis, indomethacin, 


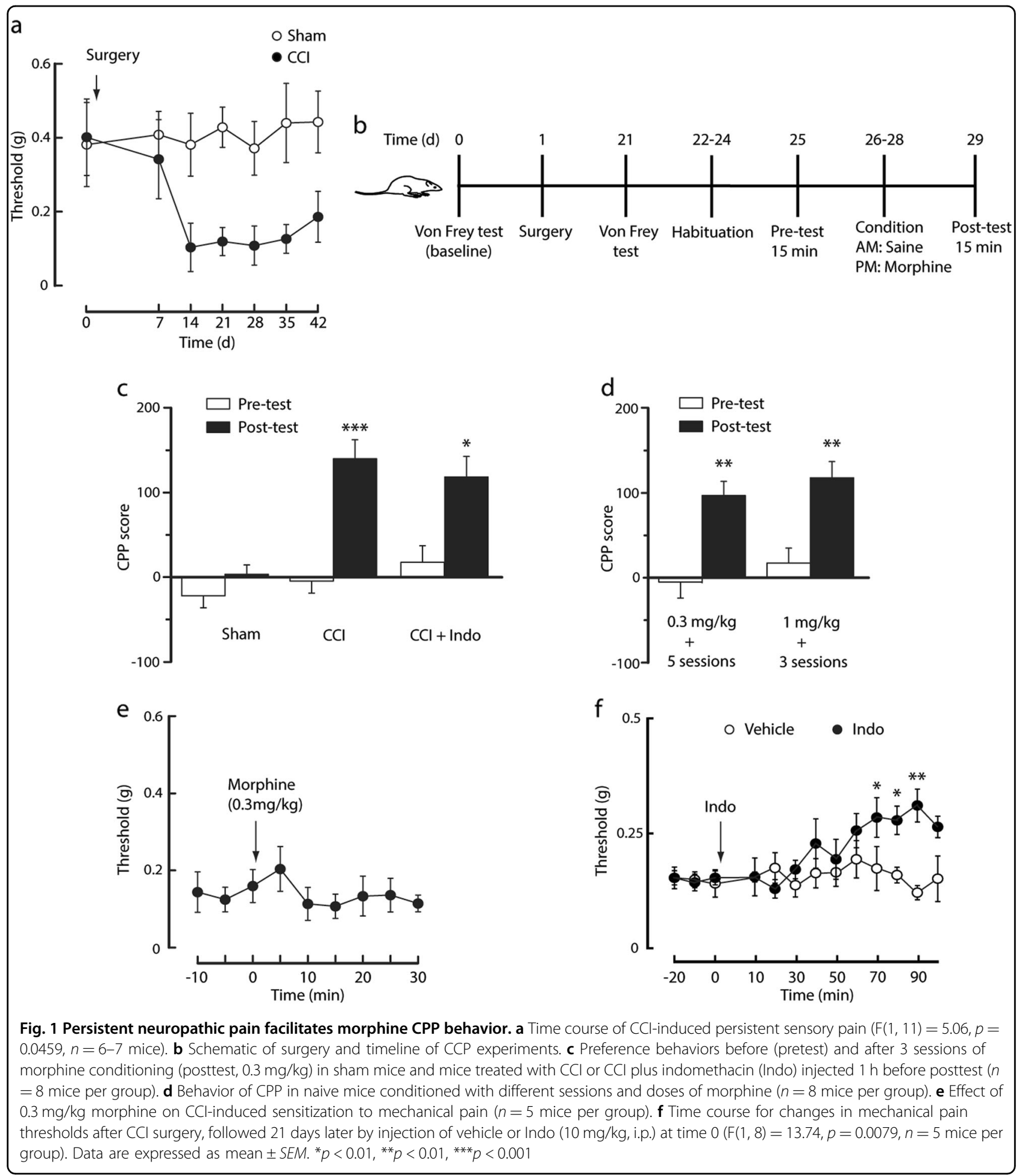

a non-opioid analgesic agent, was used to inhibite the pain before each morphine conditioning (Fig. 1f). As expected, indomethacin pretreatment $1 \mathrm{~h}$ before each session of morphine paring did not significantly alter the facilitating effect of pain on CPP acquisition in CCI mice (Fig. 1c).
Increased mPFC CRF neuronal activity contributes to morphine rewarding facilitation

Given the role of mPFC CRF signaling in drug addiction $^{24}$, we wondered whether mPFC CRF neuronal plasticity participates in neuropathic pain-induced hypersensitivity to morphine reward. To visualize CRF 


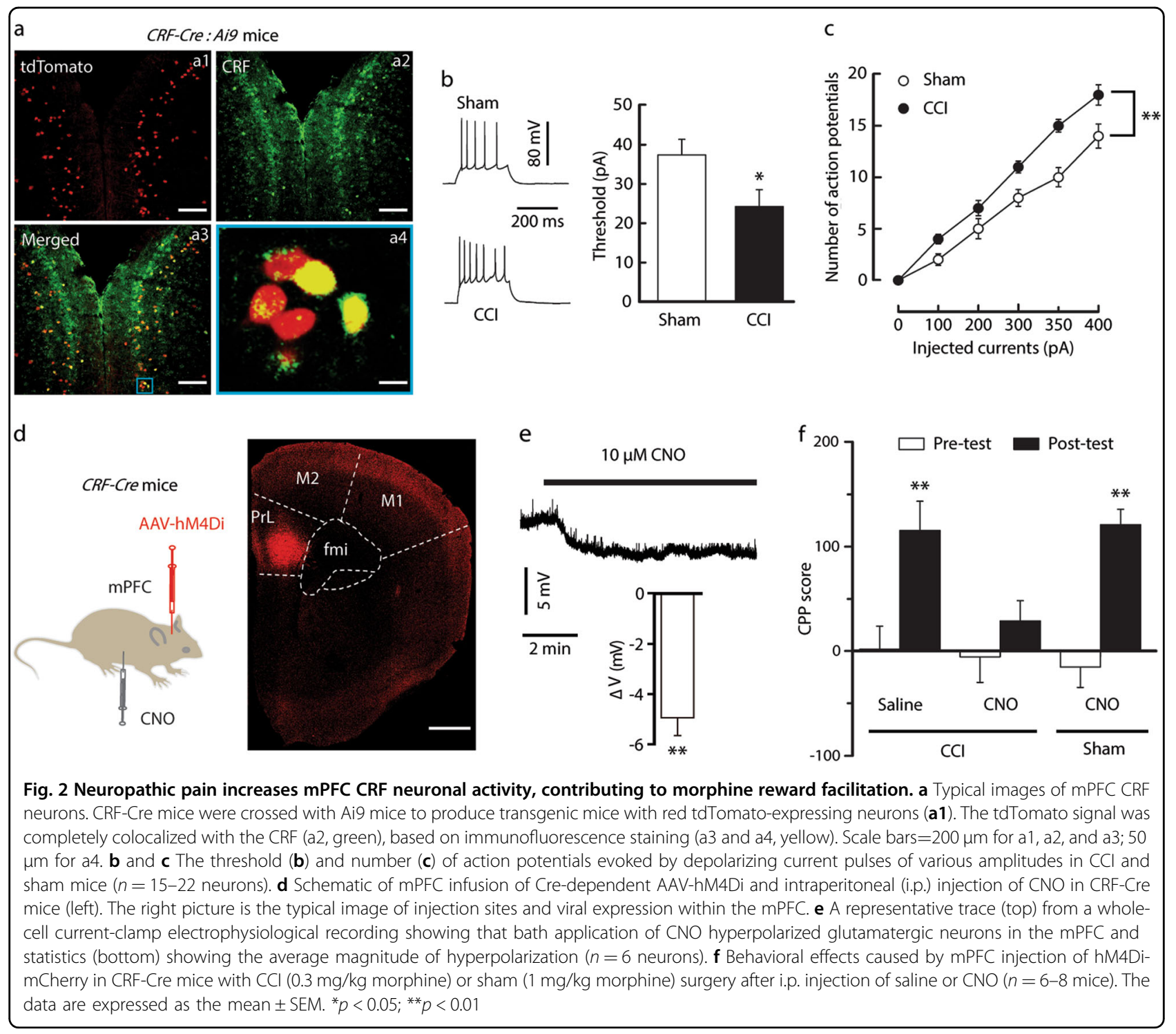

neurons for whole-cell recordings in slices, CRF-Cre mice were crossed with $A i 9$ (RCL-tdT) mice to produce transgenic mice with red tdTomato-expressing CRF (CRF-tdT). We found numerous CRF- $t d T$ neurons in the mPFC (Fig. 2a). In response to a series of current injections, compared with sham CRF-tdT mice, we found a decrease in the threshold to elicit a spike in $\mathrm{mFPC}$ $C R F-t d T$ neurons from CCI mice on day 21 after surgery and an increase in the spike number (Fig. 2b, c). Given the enhanced mPFC CRF neuronal activity in neuropathic pain, we then asked whether the inhibition of mPFC CRF neurons could restore the morphine response. Using Cre-inducible designer receptors that are exclusively inhibited by designer drugs (DREADD), system-Gi-protein-coupled receptor hM4Di and its ligand clozapine- $N$-oxide $(\mathrm{CNO}, 3 \mathrm{mg} / \mathrm{kg})^{35}$, selectively to inhibit mPFC CRF neurons in CRF-Cre mice with CCI surgery (Fig. 2d, e), we found that the CPP behavior induced by three sessions of $0.3 \mathrm{mg} / \mathrm{kg}$ morphine conditioning was blocked (Fig. 2f). Interestingly, the morphine CPP behavior in sham mice was not affected by chemogenetic inhibition of mPFC CRF neurons (Fig. 2f). These results indicate that the increase in mPFC CRF neuronal activity contributes to morphine reward facilitation by persistent neuropathic pain.

\section{CRF mPFC $\rightarrow$ NAC pathway contributes to morphine reward in neuropathic pain}

We next identified the MPFC CRF output circuitry mediating the behavioral response in neuropathic pain. By mPFC infusion of Cre-dependent adeno-associated virus that expressed channelrhodopsin-2 (AAV-ChR2) in CRFCre mice, we observed mCherry ${ }^{+}(\mathrm{CRF})$ cell bodies in the 


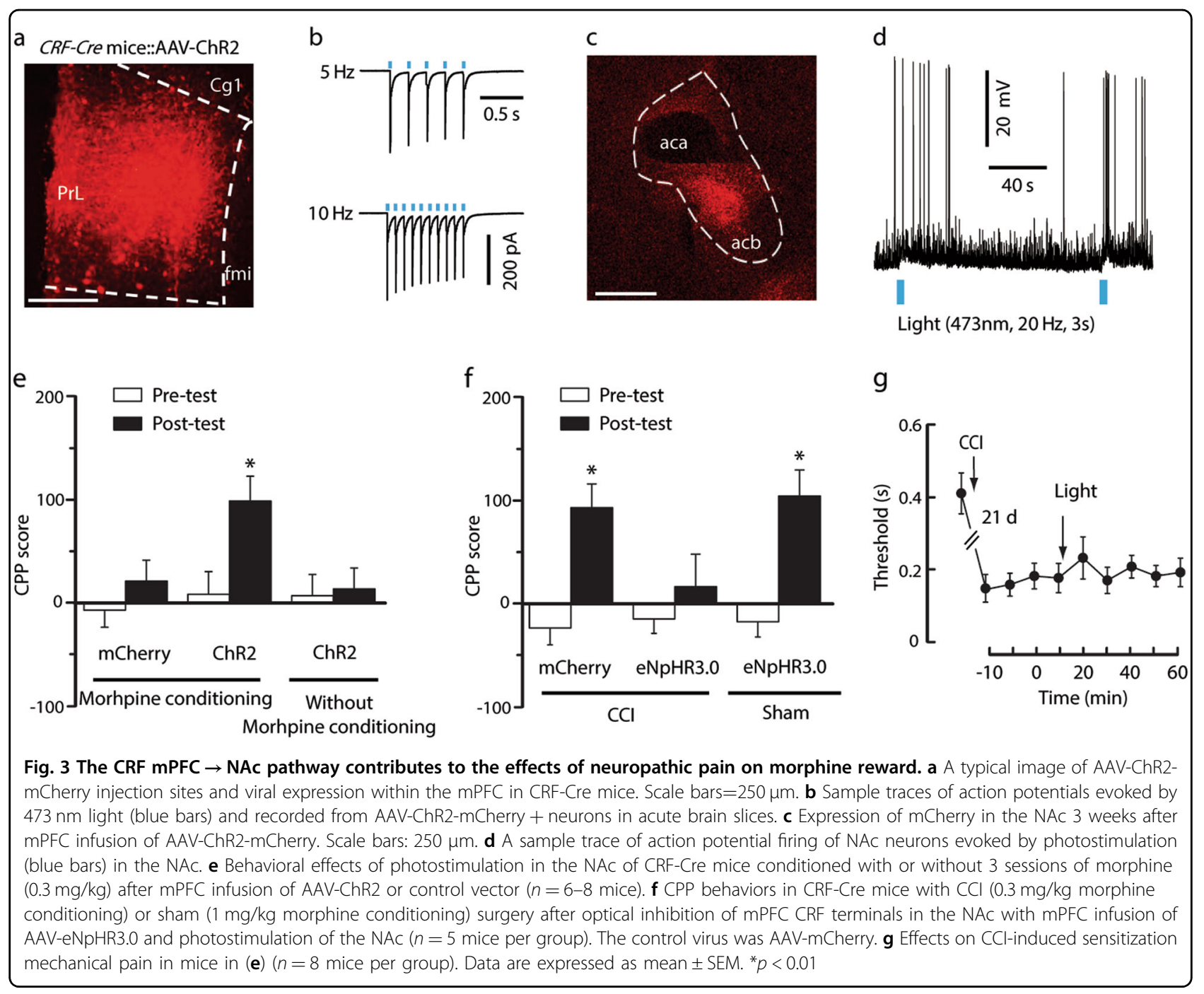

mPFC (Fig. 3a), which displayed an excitatory response to light stimulation (Fig. 3b). Meanwhile, numerous mCherry $^{+}$fibers had emerged in the NAc (Fig. 3c). Whole-cell recording showed that optical stimulation of ChR2- containing mPFC CRF terminals in the NAc elicited action potential firing in brain slices (Fig. 3d). These results suggest that NAc neurons receive direct $\mathrm{mPFC}$ CRF projections, which exert excitatory effects. Behaviorally, optical activation of the MPFC CRF terminals in the NAc showed that CPP behavior was established by subthreshold morphine conditioning $(0.3 \mathrm{mg} / \mathrm{kg})$ in sham mice (Fig. 3e). In contrast, the morphine CCP behavior was blocked by optical inhibition of the mPFC CRF terminals in the NAc of CCI mice, but not sham mice (Fig. 3f), whereas the pain sensitization was not influenced (Fig. 3g). In addition, compared with the sham mice, the NAc neuronal firing rate was increased in the CCI mice (Fig. 4a). These results establish the functional relevance of the CRF $\mathrm{mPFC} \rightarrow \mathrm{NAc}$ pathway to morphine CCP behavior under neuropathic pain conditions, but not normal conditions.

An aversive stimulus produces effects that are sufficient to drive negative reinforcement, in which a modest positive reinforcer, e.g., a low dose of morphine $(0.3 \mathrm{mg} / \mathrm{kg})$, can drive rewarding effects ${ }^{36,37}$. To examine whether activation of this mPFC CRF pathway on its own produces a place aversion, we examined the behavioral consequences after activation of the $\mathrm{mPFC} \rightarrow \mathrm{NAc}$ pathway. The CRF-Cre sham mice with mPFC injection of AAV-ChR2 were conditioned for 30 min with or without blue light stimulation in the NAc in a single daily session, which is similar to pairing saline with one chamber in the morning and pairing morphine with the other chamber in the afternoon for 5 days. The results showed no preference or averse behaviors (Fig. 3e), which suggested that activation of the mPFC CRF pathway is not sufficient to drive negative reinforcement. 


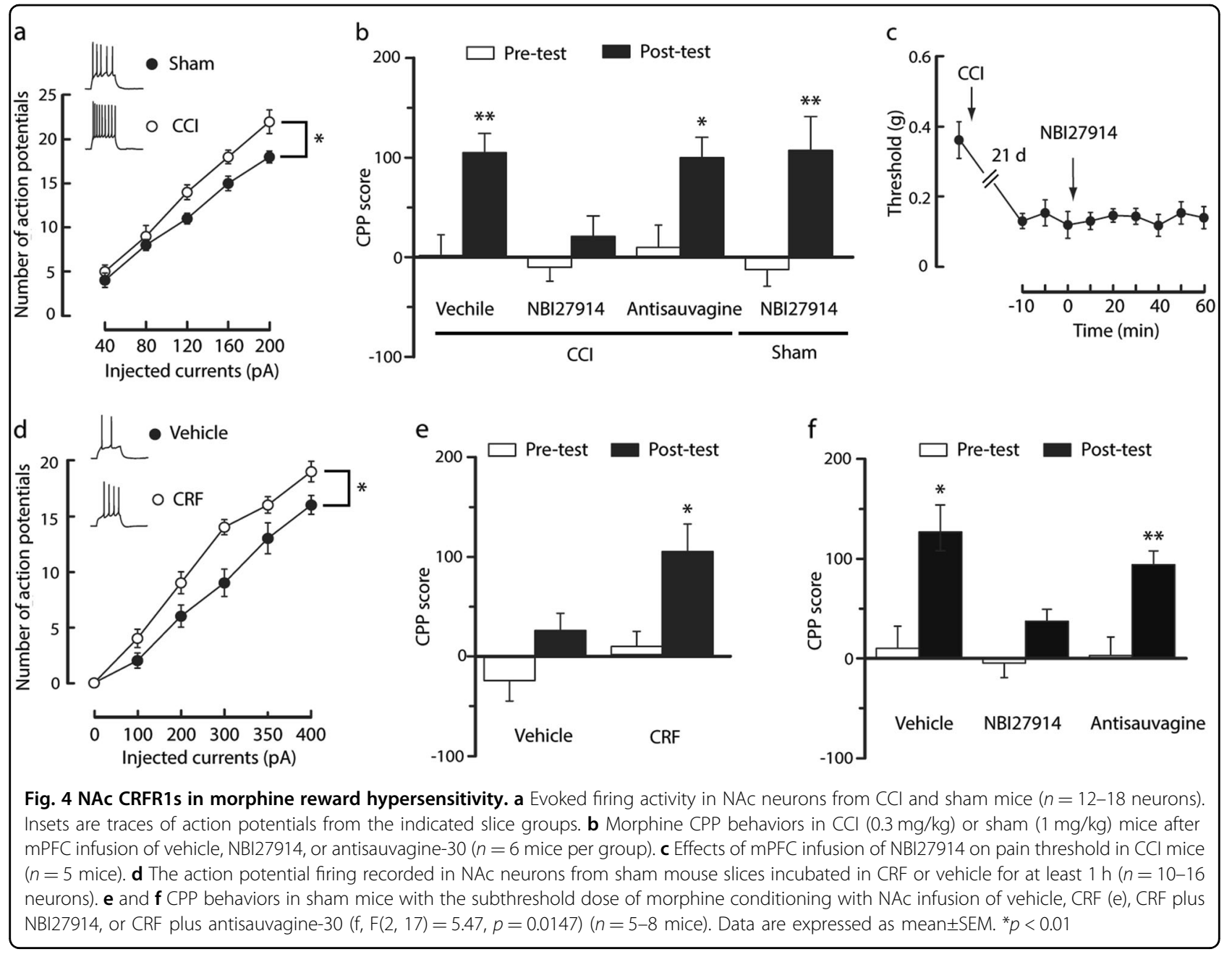

\section{NAC CRFR1s are involved in mPFC CRF actions on morphine reward in neuropathic pain}

CRF acts on both CRFR1 and CRFR2. We determined which types of CRFRs were involved in CRF actions. With the infusion of CRFR1 antagonist NBI27914 (500 ng in $200 \mathrm{nl})$ or CRFR2 antagonist antisauvagine-30 (3.6 $\mu \mathrm{g}$ in $200 \mathrm{nl}$ ) into the NAc before each morphine conditioning session in CCI mice, we found that the CPP behavior was blocked only by NBI27914 and not by antisauvagine-30 (Fig. 4b), suggesting the specific role of CRFR1 under these conditions. Notably, NBI27914 had no effects on morphine CPP behavior in sham mice (Fig. 4b). In addition, this dose of NBI27914 had no effect on CCI-induced pain sensitization (Fig. 4c).

Next, we asked whether exogenous CRF could mimic the effect produced by CCI. After treatment of sham mouse slices with CRF (100 nM) for at least $1 \mathrm{~h}$, NAc cells displayed increased action potential firing rates when compared with the cells in the vehicle-treated control slices (Fig. 4d). Furthermore, through the infusion of CRF $(1.5 \mu \mathrm{g}$ in $200 \mathrm{nl})$ into the NAc before each morphine conditioning session, CPP was established by three morphine conditioning sessions $(0.3 \mathrm{mg} / \mathrm{kg})$ in sham mice (Fig. 4e), which was inhibited by the co-infusion of NBI27914, but not by antisauvagine-30 (Fig. 4f). These results suggest that NAc CRFR1s, but not CRFR2s, are involved in $\mathrm{mPFC}$ CRF actions on neuropathic painincreased susceptibility to acquiring CPP behavior.

\section{G9a-mediated CRFR1 gene de-repression in neuropathic pain}

Notably, CRFR1 gene transcriptional activity is also altered in response to stress ${ }^{38}$. As shown in Fig. 5a, the amount of CRFR1 protein was increased by $61 \%$ in the NAc tissues taken from the CCI mice compared to that of the sham mice ( $n=6$ mice/group, $p<0.05$ ).

Studies in rodents have shown that G9a specifically catalyzes the dimethylation of histone 3 at lysine 9 (H3K9me2), which is an epigenetic marker of transcriptional repression, and regulates CRFR1 activity in both chronic pain and drug addiction ${ }^{39-41}$. We then determined whether G9a regulated the increased expression of 

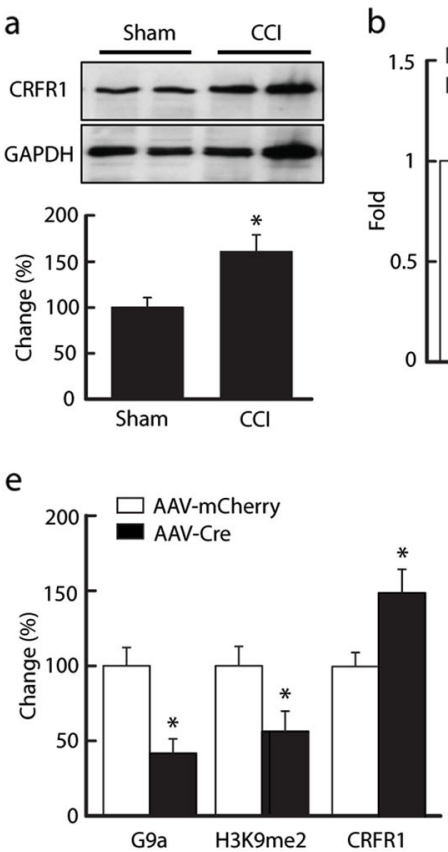

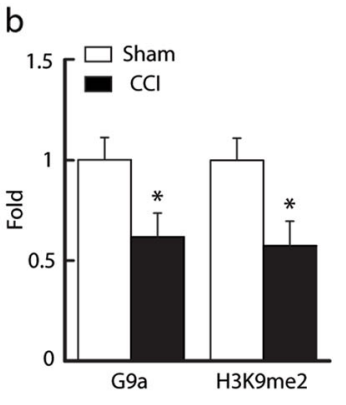

$f$

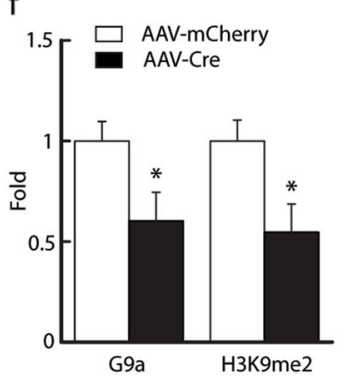

C

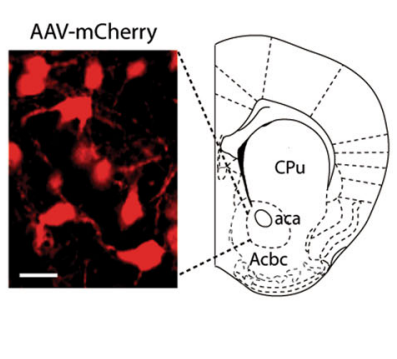

d

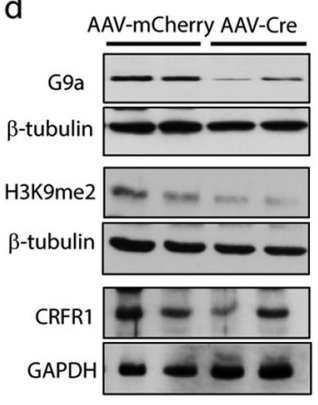

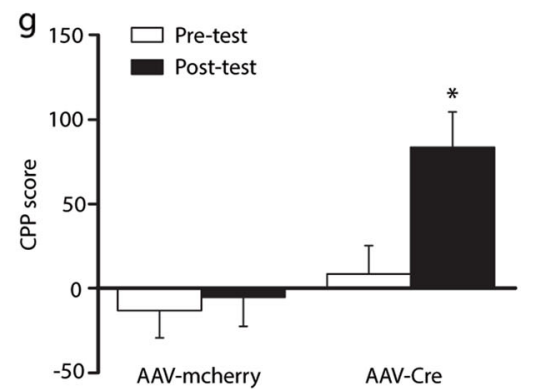

Fig. 5 G9a represses CRFR1s. a Representative Western blots (top) and summarized results (bottom) of CRFR1 protein in the NAc from CCI and sham mice ( $n=6$ mice per group). The molecular mass was $\sim 51$ kD for CRFR1s and 37 kD for GAPDH. b Normalized levels of G9a and H3K9me2 across the CRFR1 promoter region in NAc tissues from mice in a ( $n=8$ mice per group). c Representative images of expression of an AAV-Cre vector infused in the NAc from a G9a fl/fl mouse. Scale bars $=250 \mathrm{~mm}$. d, e Western blots (d) and summarized data (e) of NAc levels of G9a, H3K9me2, and CRFR1 in G9a ${ }^{f / f l}$ mice after NAc infusion of AAV-GFP (control) or AAV-Cre ( $n=6$ mice per group). $\mathbf{f}$ Normalized levels of G9a and H3K9me2 across the CRFR1 promoter region in NAc tissues from mice in (e) ( $n=6$ mice per group). $\mathbf{g}$ CPP behaviors in naïve G9a ${ }^{\mathrm{fl} / \mathrm{fl}}$ mice conditioned with $0.3 \mathrm{mg} / \mathrm{kg}$ morphine after NAc infusion of AAV-GFP (control) or AAV-Cre ( $n=8$ mice per group)

CRFR1s. Chromatin immunoprecipitation (CHIP) assays showed that the level of G9a at the CRFR1 gene promoter regions was reduced in the CCI mice (Fig. 5b; $61.8 \pm 11.8 \%$ of the sham control, $n=9$ mice per group, $p<0.05)$, and H3K9me2 changed accordingly along with G9a (Fig. 5b; $57.4 \pm 12.2 \%$ of sham control, $n=9$ mice per group, $p<$ 0.05). These results suggest that CCI decreased CRFR1 expression via a G9a-mediated epigenetic mechanism.

To characterize the function of this epigenetic regulation of behavior response, we examined morphine CCP behavior after knockdown of G9a in the NAc using an AAVEF1a-mCherry-IRES-WGA- Cre (AAV-Cre) vector (Fig.5c). As shown in Fig. 5d and e, the infusion of AAVCre into the NAc of $\mathrm{G} 9 \mathrm{a}^{\mathrm{fl} / \mathrm{fl}}$ mice significantly reduced the levels of G9a protein $(41.7 \pm 9.6 \%$ of the control, $n=6$ mice per group, $p<0.05)$ and H3K9me2 $(56.2 \pm 13.7 \%$ of the control, $n=6$ mice per group, $p<0.05)$, while the level of CRFR1 protein was increased (149.2 $\pm 15.6 \%$ of the sham control, $n=6$ mice per group, $p<0.05)$. Meanwhile, G9a and H3K9me2 occupancy on the CRFR1 promoters was reduced (Fig. 5f). Behaviorally, AAV-Creinjected $\mathrm{G} 9 \mathrm{a}^{\mathrm{fl} / \mathrm{fl}}$ mice displayed increased sensitivity to CPP behavior induced by a subthreshold dose of morphine when compared with the AAV-GFP-injected control mice (Fig. 5g).

\section{Discussion}

Circuits comprising the mPFC are essential in processing emotion, and therefore are involved in the affective component of pain and drug reward ${ }^{20,42-45}$. A comprehensive understanding regarding the functionality of painrelevant circuitry in the $\mathrm{mPFC}$ is lacking. We found that neuropathic pain increases the response to morphine reward, accompanied by increased mPFC CRF neuronal activity. Given that pain and cognitive processing are represented in overlapping regions, the mPFC circuitry may regulate the reciprocal relationship that exists between neuropathic pain and opioid reward. Previous studies have shown that GABAergic neurons in the central nucleus of the amygdala (CeA) are involved in pain facilitation of the reward response ${ }^{46}$, whereas the functional output of the CeA that produces these processes is unknown. It has been shown that pain-related hyperactivity in the amygdala leads to the deactivation of the mPFC and decision-making deficits, suggesting that functional interactions between the amygdala and PFC are important for emotional learning and behavior ${ }^{45,47}$. Thus, it is possible that the altered mPFC activity is triggered by pain-modulatory regions, such as the amygdala ${ }^{48,49}$, to produce morphine-reward facilitation. Of particular interest is that persistent inflammation does not change 
the amount of self-administered morphine in rats, but increases the morphine-seeking behavior after morphine withdrawal ${ }^{50}$. Both of these factors work to implicate the long-lasting effect of pain on the risk of opioid misuse. The subtle difference may result from differences in the species (mice vs. rats) and the behavioral paradigms (CPP vs. self-administration).

Increased functional connectivity between the NAc and the prefrontal cortex, which takes place through corticostriatal-pallidal-thalamic loops, is predictive of the transition from acute to chronic pain ${ }^{21,51,52}$, indicating the clinical significance of this pathway. Our finding that mPFC CRF neurons project to the NAc to exert excitatory effects supports this notion. Interestingly, the inhibition of the CRF $\mathrm{mPFC} \rightarrow \mathrm{NAc}$ pathway blocked neuropathic pain effects on morphine reward, whereas it has no effect on pain sensitization. In fact, until recently, NAc was thought to encode salience for pain, while NAc valuation of acute analgesia was distorted in chronic pain patients ${ }^{53}$. Our results suggest that, at least for the CRF system, the $\mathrm{mPFC} \rightarrow$ NAc pathway is involved in morphine susceptibility, but not pain relief, under neuropathic pain conditions. In addition, a previous study reported that when pain was acutely inhibited by analgesic agents, the acquisition of morphine CPP was still facilitated in inflammatory pain animals ${ }^{46,54}$. Consistent with this finding, when we used a non-opioid analgesic agent to inhibit pain before each morphine conditioning, the CPP behavior was not affected in CCI mice. Furthermore, our results showed that $0.3 \mathrm{mg} / \mathrm{kg}$ morphine, which induced CCP in CCI mice, had no effect on CCI-induced pain sensitization. These results indicate that the susceptible CPP response is due more to a heightened rewarding effect of morphine than to morphine's inhibition of acute pain. Notably, in normal mice, inhibition of the MPFC $\rightarrow$ NAc pathway had no effects on morphine CPP behavior, whereas activation of this pathway facilitated CPP acquisition. These results suggest that persistent pain- induced CPP occurs through a specific mechanism.

It is presumed that pain is an inherent stressor ${ }^{55}$. CRF is thought to be a key molecular link between the behavioral effects of stress and drugs of abuse. Although the hypothalamic-pituitary-adrenal (HPA) axis, a source of CRF, plays a fundamental role in regulating the behavioral and neuroendocrine responses to stressors, whereas a previous study found CCI did not affect indices of basal HPA axis activity ${ }^{56}$. This suggests that the role of CRF in pain modulation may take place beyond the HPA. Although the CRF antibody is not ideal for CRF immunoreactivity in different species, including mice, rats and primates, we found that the mPFC is a CRF-rich brain region in mice. Persistent neural injury enhanced mPFC CRF neuronal activity, and chemogenetic inhibition of these neurons reversed $\mathrm{CCI}$ - induced morphine CPP facilitation. These results indicate that $\mathrm{MPFC}$ CRF is a critical modulator linking neuropathic pain and morphine reward hypersensitivity, which could extend beyond the glutamatergic and GABAergic systems in the mPFC. This notion is supported by the finding that the functional interactions between dopamine and CRF systems regulate drug abuse independently of the effects on the pituitary systems $^{27,30}$. In addition, the amygdala CRF contributes to pain chronification and mediates the affective component of pain ${ }^{31,57}$. Whether the amygdala CRF is involved in persistent pain-increased morphine susceptibility needs further investigated.

Both the CRF1 and CRF2 receptors are involved in generating emotional pain responses ${ }^{29}$. The current study shows that mPFC CRF-mediated morphine reward hypersensitivity under neuropathic pain conditions develops through NAc CRFR1s, but not CRFR2s, which is consistent with previous findings that the activation of CRFR1 in the NAc induces a positive affective state ${ }^{2}$. In addition, the optical inhibition of mPFC CRF terminals in the NAc reversed the morphine response to neuropathic pain, but not pain sensitization, confirming that the CRFCRF1 system in the mPFC $\rightarrow$ NAc circuit is not involved in the etiology and maintenance of CCI-induced allodynia. Of note, CRF/CRFR1 signaling has a complicated physiological effect. For example, CRF has both analgesic and anti-hyperalgesic effects in rodent pain models ${ }^{28}$.

Numerous types of epigenetic modifications within the brain's reward circuitry in animal models of chronic pain and drug addiction have been investigated ${ }^{58,59}$. For example, chronic morphine downregulates G9a in the NAc, and G9a overexpression promotes analgesic tolerance and withdrawal ${ }^{39,40}$. The present study provides original evidence that G9a, through transcriptional derepression of CRFR1 via deceased binding of H3K9me2 at the CRFR1 gene promoter, promotes CCP behavior. This finding is in general agreement with the previous finding that the downregulation of G9a in the CeA by persistent inflammatory pain contributes to the preference behavior of morphine reward ${ }^{54}$. Furthermore, stress reduces the level of H3K9 trimethylation at the CRFR1 gene promoter $^{38}$. Thus, neuropathic pain induces the dual enhancement of CRFR1 expression and mPFC CRF inputs in the NAc in a manner that is persistent and reliable enough to maintain mPFC-NAc circuitry maladaptation, generating susceptibility to morphine reward. Although the levels of CRFR1 do not govern the dominant actions of CRF, our results suggest that CRFR1 is a potential cofactor to the physiological effects of CRF.

Collectively, the present study defines a specific $\mathrm{mPFC} \rightarrow$ NAc CRF neuronal circuit through which persistent neuropathic pain increases the response to morphine reward. Central to this process is a circuit mechanism that involves the increased activity in NAc- 
projecting mPFC CRF neurons after chronic nerve injury. Meanwhile, the increased mPFC CRF input exerts excitatory effects on the NAc via CRFR1s, which is upregulated by a G9a-H3K9me2-mediated epigenetic mechanism. This convergence of regulating mechanisms provides a circuitry and molecular basis for understanding neuropathic pain-facilitated opioid reward effects.

\section{Acknowledgements}

We thank Yupeng Yang for providing the Ai9 mice. We thank Jiangning Zhou for providing the CRF-Cre mouse strain. This work was supported by the National Natural Science Foundation of China (Grant No. 31600851 and 91732303), 973 Program (Grant No. 2014CB548100), and the Strategic Priority Research Program of the Chinese Academy of Sciences (Grant No. XDB02010000).

\section{Author details}

${ }^{1}$ Key Laboratory of Brain Function and Disease of Chinese Academy of Science, Department of Biophysics and Neurobiology, University of Science and Technology of China, Hefei 230027, China. ${ }^{2}$ Institute of Health Sciences and technology, School of Life Sciences, Anhui University, Hefei, Anhui 2300601 China. ${ }^{3}$ Department of Anesthesiology and Department of Pain Management, The First Affiliated Hospital of Anhui Medical University, Hefei, Anhui 230022 China. ${ }^{4}$ Department of Psychology, Anhui Mental Health Center, Hefei, Anhui 230022, China

\section{Conflict of interest}

The authors declare that they have no conflict of interest.

\section{Publisher's note}

Springer Nature remains neutral with regard to jurisdictional claims in published maps and institutional affiliations.

Received: 20 March 2018 Accepted: 4 April 2018

Published online: 21 May 2018

\section{References}

1. Lutz, J., Gross, R., Long, D. \& Cox, S. Predicting risk for opioid misuse in chronic pain with a single-item measure of catastrophic thinking. J. Am. Board Fam. Med. 30, 828-831 (2017).

2. Passik, S. D. \& Kirsh, K. L. Addictions in pain clinics and pain treatment. Ann. N. Y. Acad. Sci. 1216, 138-143 (2011).

3. Smith, H. S. Opioids and neuropathic pain. Pain. Physician 15, ES93-ES110 (2012).

4. Voon, P., Karamouzian, M. \& Kerr, T. Chronic pain and opioid misuse: a review of reviews. Subst. Abus. Treat. Prev. Policy 12, 36 (2017).

5. Ballantyne, J. C. \& LaForge, K. S. Opioid dependence and addiction during opioid treatment of chronic pain. Pain 129, 235-255 (2007).

6. Soelberg, C. D. et al. The US opioid crisis: current federal and state legal issues. Anesth. Analg. 125, 1675-1681 (2017).

7. Woolf, C. J. \& Hashmi, M. Use and abuse of opioid analgesics: potential methods to prevent and deter non-medical consumption of prescription opioids. Curr. Opin. Investig. Drugs 5, 61-66 (2004).

8. Volkow, N., Benveniste, H. \& McLellan, A. T. Use and misuse of opioids in chronic pain. Annu. Rev. Med. 69, 451-465 (2017).

9. Koob, G. F. \& Le Moal, M. Review. Neurobiological mechanisms for opponent motivational processes in addiction. Philos. Trans. R. Soc. Lond. B Biol. Sci. $\mathbf{3 6 3}$ 3113-3123 (2008).

10. Hyman, S. E., Malenka, R. C. \& Nestler, E. J. Neural mechanisms of addiction: the role of reward-related learning and memory. Annu. Rev. Neurosci. 29, 565-598 (2006).

11. Lumley, M. A. et al. Pain and emotion: a biopsychosocial review of recent research. J. Clin. Psychol. 67, 942-968 (2011).

12. Simons, L. E., Elman, I. \& Borsook, D. Psychological processing in chronic pain: a neural systems approach. Neurosci. Biobehav. Rev. 39, 61-78 (2014).
13. Upadhyay, J. et al. Alterations in brain structure and functional connectivity in prescription opioid-dependent patients. Brain: J. Neurol. 133, 2098-2114 (2010). Pt 7

14. Elman, I. \& Borsook, D. Common brain mechanisms of chronic pain and addiction. Neuron 89, 11-36 (2016).

15. Torkaman-Boutorabi, A., Sheidadoust, H. Hashemi-Hezaveh, S. M. \& Zarrindast, M. R. Influence of morphine on medial prefrontal cortex alpha2 adrenergic system in passive avoidance learning in rats. Pharmacol. Biochem. Behav. 133, 92-98 (2015).

16. Bland, S. T. et al. Stressor controllability modulates stress-induced dopamine and serotonin efflux and morphine-induced serotonin efflux in the medial prefrontal cortex. Neuropsychopharmacology 28, 1589-1596 (2003).

17. Zhu, Y., Wienecke, C. F., Nachtrab, G. \& Chen, X. A thalamic input to the nucleus accumbens mediates opiate dependence. Nature 530, 219-222 (2016).

18. Scofield, M. D. et al. The nucleus accumbens: mechanisms of addiction across drug classes reflect the importance of glutamate homeostasis. Pharmacol. Rev. 68, 816-871 (2016)

19. Chang, P. C. et al. Role of nucleus accumbens in neuropathic pain: linked multi-scale evidence in the rat transitioning to neuropathic pain. Pain 155 1128-1139 (2014)

20. Metz, A. E., Yau, H. J., Centeno, M. V., Apkarian, A. V. \& Martina, M. Morphological and functional reorganization of rat medial prefrontal cortex in neuropathic pain. Proc. Natl Acad. Sci. USA 106, 2423-2428 (2009).

21. Baliki, M. N. et al. Corticostriatal functional connectivity predicts transition to chronic back pain. Nat. Neurosci. 15, 1117-1119 (2012).

22. Regev, L. \& Baram, T. Z. Corticotropin releasing factor in neuroplasticity. Front Neuroendocrinol. 35, 171-179 (2014).

23. Inda, C., Armando, N. G., Dos Santos Claro, P. A. \& Silberstein, S. Endocrinology and the brain: corticotropin-releasing hormone signaling. Endocr. Connect. $\mathbf{6}$ R99-R120 (2017).

24. Zorrilla, E. P., Logrip, M. L. \& Koob, G. F. Corticotropin releasing factor: a key role in the neurobiology of addiction. Front. Neuroendocrinol. 35, 234-244 (2014).

25. Koob, G. F. The role of CRF and CRF-related peptides in the dark side of addiction. Brain Res. 1314, 3-14 (2010).

26. Koob, G. F. \& Zorrilla, E. P. Neurobiological mechanisms of addiction: focus on corticotropin-releasing factor. Curr. Opin. Investig. Drugs 11, 63-71 (2010).

27. Park, P. E. et al. Chronic CRF1 receptor blockade reduces heroin intake escalation and dependence-induced hyperalgesia. Addict. Biol. 20, 275-284 (2015).

28. Lariviere, W. R. \& Melzack, R. The role of corticotropin-releasing factor in pain and analgesia. Pain 84, 1-12 (2000).

29. Fu, Y. \& Neugebauer, V. Differential mechanisms of CRF1 and CRF2 receptor functions in the amygdala in pain- related synaptic facilitation and behavior. J. Neurosci. 28, 3861-3876 (2008).

30. Nijsen, M., Ongenae, N., Meulemans, A. \& Coulie, B. Divergent role for CRF1 and CRF2 receptors in the modulation of visceral pain. Neurogastroenterol. Motil. 17, 423-432 (2005).

31. Andreoli, M., Marketkar, T. \& Dimitrov, E. Contribution of amygdala CRF neurons to chronic pain. Exp. Neurol. 298, 1-12 (2017).

32. Alexander, G. M. et al. Remote control of neuronal activity in transgenic mice expressing evolved G protein-coupled receptors. Neuron 63, 27-39 (2009).

33. Zhang, Z., Cai, Y. Q., Zou, F., Bie, B. \& Pan, Z. Z. Epigenetic suppression of GAD65 expression mediates persistent pain. Nat. Med. 17, 1448-1455 (2011).

34. Mogil, J. S. Animal models of pain: progress and challenges. Nat. Rev. Neurosci. 10, 283-294 (2009).

35. Armbruster, B. N., Li, X., Pausch, M. H., Herlitze, S. \& Roth, B. L. Evolving the lock to fit the key to create a family of $G$ protein-coupled receptors potently activated by an inert ligand. Proc. Natl Acad. Sci. USA 104, 5163-5168 (2007).

36. Koob, G. F. Negative reinforcement in drug addiction: the darkness within. Curr. Opin Neurobiol. 23, 559-563 (2013).

37. Wise, R. A. \& Koob, G. F. The development and maintenance of drug addiction. Neuropsychopharmacology 39, 254-262 (2014).

38. Wan, Q. et al. Histone modifications of the Crhr1 gene in a rat model of depression following chronic stress. Behav. Brain. Res. 271, 1-6 (2014).

39. Maze, I. et al. Essential role of the histone methyltransferase G9a in cocaineinduced plasticity. Science 327, 213-216 (2010).

40. Sun, $\mathrm{H}$. et al. Morphine epigenomically regulates behavior through alterations in histone H3 lysine 9 dimethylation in the nucleus accumbens. J. Neurosci. 32 , 17454-17464 (2012)

41. Laumet, G. et al. G9a is essential for epigenetic silencing of $\mathrm{K}(+)$ channel genes in acute-to-chronic pain transition. Nat. Neurosci. 18, 1746-1755 (2015). 
42. Tzschentke, T. M. Pharmacology and behavioral pharmacology of the mesocortical dopamine system. Prog. Neurobiol. 63, 241-320 (2001).

43. Nasif, F. J., Sidiropoulou, K., Hu, X. T. \& White, F. J. Repeated cocaine administration increases membrane excitability of pyramidal neurons in the rat medial prefrontal cortex. J. Pharmacol. Exp. Ther. 312, 1305-1313 (2005).

44. Apkarian, A. V. et al. Neural mechanisms of pain and alcohol dependence. Pharmacol. Biochem. Behav. 112, 34-41 (2013).

45. Ji, G. \& Neugebauer, V. Pain-related deactivation of medial prefrontal cortical neurons involves mGluR1 and GABA(A) receptors. J. Neurophysiol. 106, 2642-2652 (2011).

46. Zhang, Z. et al. Persistent pain facilitates response to morphine reward by downregulation of central amygdala GABAergic function. Neuropsychopharmacology 39, 2263-2271 (2014).

47. Ji, G. et al. Cognitive impairment in pain through amygdala- driven prefrontal cortical deactivation. J. Neurosci.: Off. J. Soc. Neurosci. 30, 5451-5464 (2010).

48. Little, J. P. \& Carter, A. G. Synaptic mechanisms underlying strong reciprocal connectivity between the medial prefrontal cortex and basolateral amygdala. J. Neurosci.: Off. J. Soc. Neurosci. 33, 15333-15342 (2013).

49. Sakaki, M., Nga, L. \& Mather, M. Amygdala functional connectivity with medial prefrontal cortex at rest predicts the positivity effect in older adults' memory. J. Cogn. Neurosci. 25, 1206-1224 (2013).

50. Hou, Y. Y., Cai, Y. Q. \& Pan, Z. Z. Persistent pain maintains morphine-seeking behavior after morphine withdrawal through reduced MeCP2 repression of GluA1 in rat central amygdala. J. Neurosci. 35, 3689-3700 (2015).
51. Baliki, M. N. et al. Chronic pain and the emotional brain: specific brain activity associated with spontaneous fluctuations of intensity of chronic back pain. J. Neurosci.: Off. J. Soc. Neurosci. 26, 12165-12173 (2006).

52. Baliki, M. N., Geha, P. Y., Fields, H. L. \& Apkarian, A. V. Predicting value of pain and analgesia: nucleus accumbens response to noxious stimuli changes in the presence of chronic pain. Neuron 66, 149-160 (2010).

53. Becerra, L. \& Borsook, D. Signal valence in the nucleus accumbens to pain onset and offset. Eur. J. Pain. 12, 866-869 (2008).

54. Zhang, Z. et al. MeCP2 repression of G9a in regulation of pain and morphine reward. J. Neurosci.: Off. J. Soc. Neurosci. 34, 9076-9087 (2014).

55. Vierck, C. J., Green, M. \& Yezierski, R. P. Pain as a stressor: effects of prior nociceptive stimulation on escape responding of rats to thermal stimulation. Eur. J. Pain. 14, 11-16 (2010).

56. Bomholt, S. F., Mikkelsen, J. D. \& Blackburn-Munro, G. Normal hypothalamopituitary-adrenal axis function in a rat model of peripheral neuropathic pain. Brain Res. 1044, 216-226 (2005).

57. Rouwette, T. et al. Experimental neuropathy increases limbic forebrain CRF. Eur. J. Pain. 16, 61-71 (2012).

58. Liang, L., Lutz, B. M., Bekker, A. \& Tao, Y. X. Epigenetic regulation of chronic pain. Epigenomics 7, 235-245 (2015).

59. Nestler, E. J. Epigenetic mechanisms of drug addiction. Neuropharmacology 76, 259-268 (2014). Pt B. 